\title{
Déterminisme paléoécologique des écosystèmes actuels du Haut-Jura, en relation avec la fracturation des roches
}

\author{
M Gaiffe, S Bruckert \\ Laboratoire de pédologie université de Franche-Comté, place Leclerc, \\ 25030 Besançon Cedex, France
}

(Reçu le 29 avril 1991; accepté le 28 juin 1991)

\begin{abstract}
Résumé - L'étude en parallèle des groupements végétaux, des sols et des interfaces sols-roches, a montré que les écosystèmes étaient étroitement dépendants des modes de fracturation (densité de fracture, taille des fragments) et des propriétés (système poral, conductivité hydraulique) des bancs calcaires à l'affleurement dans la haute-chaîne jurassienne (800-1 $700 \mathrm{~m}$ ). Aux étages montagnard et subalpin, 9 groupements végétaux et 3 types de sols ont été associés aux 3 types de bacs qui caractérisent le contexte géologique karstique : des lapiez (blocs séparés par de larges fractures); des calcaires concassés (bancs éclatés selon un réseau serré de fines fractures); des bancs fragmentés en dalles séparées par d'étroites fractures. Le système poral assure l'évacuation des eaux gravitaires avec des dynamiques et des effets propres à chaque type de banc. Dans des milieux qui reçoivent les mêmes précipitations, le substratum géologique, par le biais de la fracturation, de la géométrie des fragments et de l'organisation à l'interface sol-roche, crée des flux hydriques et des flux de calcium qui différencient les écosystèmes. Compte tenu du fait que la fracturation a été surtout d'origine tectonique, le déterminisme de l'aptitude à la cassure des roches a été recherché dans la composition des calcaires. Les teneurs en calcite, en dolomie, en résidu insoluble et en matière organique de ce résidu, ont différencié significativement les types de bancs, indiquant ainsi que la différenciation de composition s'est opérée précocement au moment de la sédimentation. La morphologie actuelle des bancs a donc été attribuée à 2 séries d'événements, de nature sédimentologique, et de nature orogénique. Les premiers relient l'écologie du bassin sédimentaire de l'ère secondaire à l'expression actuelle des écosystèmes.
\end{abstract}

écosystème montagnard / fracturation des roches / composition des roches / effet roche / paléo-écologie des sédiments calcaires / karst du Jura

Summary - Impact of past sediment ecology on rock fracturation and distribution of current ecosystems (Jura, France). Differences in the fracture type of limestone rocks have resulted in the formation of several main plant soil ecosystems in the montane and subalpine zones of the Jura $(800-1700 \mathrm{~m})$. The sites were on stable landscape with slope $<5 \%$. Locations were chosen to reflect the variation in physical properties of the bedrock and lithic contact. The rock fractures (densities and size), the shape and size of the fragments and the hydraulic conductivities were described and analyzed to characterize the 3 main bedrocks in the areas studied (table I): 1), lapiaz, ie, large rock fragments separated from each other by wide fractures (figs 1-2), "broken" rocks traversed by numerous fine fractures (figs 2-3), paving-stones crossed by infrequent narrow fractures (fig 3). The effects of rock fracturing on vegetation (table II) and soil formation were significant in reference to porosity and permeability relationships (figs 6-7). Under similar precipitation, meteoric waters flow through the soil and porosity is relative to fracture systems (figs 4,5 ). The weathering of cobbles in the soil profiles and along the lithic contacts maintains different soil solution Ca levels and is an important variable in soil and ecosystem formation (table III). 
Regarding the regional orogenic phases and the tectonic origin of the fractures, we postulate that the different types of fracturation originated from the different chemical and mineralogic composition of the rocks. Significant differences exist in both the calcite and dolomite content, in the insoluble residue content (table IV) and in the percentage of organic matter of the carbonate-free residues (table $\mathrm{V}$, fig 8). The results indicate that the differences in rock composition arose early at about the period of sedimentation. The origin of the differentiation might be due to the sedimentation conditions and environment (fig 9). It is concluded that the present-day plant soil ecosystems may be related to the marine sediment environments of the Jurassic period (fig 10).

montane ecosystem / rock fracturing / rock composition / rock effect / limestone sediment palaeoecology / Jura karst

\section{INTRODUCTION}

Aux étages montagnard et subalpin du Jura, la distribution des écosystèmes est apparue depuis longtemps étroitement dépendante des propriétés physiques des roches (Thurmann, 1849; Beguin, 1972). Les faciès du Jurassique supérieur sont en effet constitués par $300 \mathrm{~m}$ de roches solubles, très fracturées et très perméables en grand (Itty et Vintaer, 1987). La fracturation comprend d'importantes failles qui découpent les terrains en compartiments. Ces derniers sont eux-mêmes fracturés, mais avec des densités et des modalités très différentes qui s'observent à l'affleurement (juxtaposition des bancs) et en profondeur dans les carrières (stratification). Les modes de fracturation des roches présentent des caractéristiques clairement définies par rapport auxquelles la réponse des sols et des groupements végétaux est nette (Gaiffe et Schmitt, 1980; Bruckert et Gaiffe, 1980; Michalet, 1982; Michalet et Bruckert, 1986). La couverture pédologique et les roches sousjacentes constituent en effet, par leur réseau poral, des éléments interdépendants dans une même unité hydrologique. Le fonctionnement de ces systèmes dits de subsurface (Bruckert et Gaiffe, 1990) règle les transferts de matières et détemine, dans une large mesure, les associations de plantes.
Les bancs de calcaires à grain fin (calcaires micritiques) du Jurassique supérieur (ltty et Vintaer, 1987) qui s'étagent dans le massif selon une succession de plateaux subhorizontaux, se classent en 3 types morphologiques principaux (Aubert, 1969) :

- des lapiez, disjoints par de larges fractures en blocs tabulaires de l'ordre du $\mathrm{m}^{3}$;

- des calcaires concassés (Bruckert et Gaiffe, 1980), constitués de bancs traversés d'un réseau dense de fractures séparant des cailloux d'ordre décimétrique;

- des dalles ou bancs modérément fracturés en larges pavés jointifs.

Ces modes totalement différents de fracturation ne procèdent pas de l'altération. Cette dernière est en effet essentiellement de type pelliculaire (Lamouroux, 1972) dans les faciès concernés et ne ferait qu'agrandir des fissures préexistantes (Aubert, 1969).

La fracturation par le gel qui agit sur les calcaires gélifs (poreux, à texture grossière) comme ceux du Jurassique moyen (Mathieu et Petiot, 1973), ne s'applique pas aux calcaires du Jurassique supérieur de la Haute-Chaîne parce qu'ils sont compacts, sublithographiques, denses, non poreux, bien cimentés et cohérents, à texture fine et homogène (Villain, 1965).

Les fractures sont surtout d'origine tectonique et sont soit l'expression en surface de cassures anciennes du socle profond, 
soit le résultat de plusieurs poussées orogéniques (Forestier et Verez, 1978; Chauve, 1990). Cependant, lapiez, bancs concassés et dalles coexistent côte à côte, démontrant que la réponse des roches aux actions tectoniques a été différente et donc que l'explication orogénique reste insuffisante.

Plusieurs chercheurs ont observé que la densité de fissuration était influencée dans une certaine mesure par la lithologie et par la position relative des bancs dans les falaises (facteur type de banc) (Itty et Vintaer, 1987; Salperwyck, 1990). Mais aucune recherche sur l'influence éventuelle de la composition chimique des bancs n'a été effectuée. Or, il est connu que les propriétés d'une substance pure (ici $\mathrm{CaCO}_{3}$ ) sont modifiées par la présence d'une impureté. Pour cette raison, on a émis l'hypothèse que la composition des calcaires pouvait rendre compte dans une certaine mesure de la réponse différente des roches de la Haute-Chaîne aux poussées orogéniques.

Les objectifs poursuivis dans cette étude ont donc été de présenter:

- les caractéristiques et propriétés des bancs;

- les éléments de définition des écosystèmes;

- l'impact des transferts hydriques sur le fonctionnement des sols et des écosystèmes;

- les données analytiques qui différencient les types de bancs calcaires.

\section{MATÉRIEL ET MÉTHODES}

Les terrains étudiés s'étagent de 850 à $1700 \mathrm{~m}$ d'altitude dans l'ensemble du massif du Jura franco-suisse et concernent les calcaires micritiques séquaniens et kimmeridgiens qui s'étendent sur tout l'arc jurassien sous forme de plateaux individualisés (anticlinaux coffrés). Ces surfaces subplanes, tectonisées pendant la phase orogénique pontienne, ont été soumises aux érosions karstiques (Gaiffe et Bruckert, 1985 ) et, périodiquement, aux érosions glaciaires (Campy, 1982). Les actions érosives ont souvent remis à nu la plupart des substrats géologiques, entraînant le caractère dépendant marqué des sols et de la végétation vis-à-vis des roches. Les sites d'étude occupent des positions topographiques stables avec des pentes inférieures à $5 \%$. Les écosystèmes ont évolué sous l'effet de climats tempérés froids et pluvieux de type montagnard (850-1 $400 \mathrm{~m}$ ) et subalpin (1 400-1 $700 \mathrm{~m}$ ). Les précipitations moyennes annuelles s'élèvent de 1300 à 2200 $\mathrm{mm}$, les températures moyennes annuelles s'abaissent de 8 à $3^{\circ} \mathrm{C}$. L'excédent des précipitations sur l'évapotranspiration potentielle (drainage climatique) reste élevé toute l'année.

Au cours de travaux préalables à cette étude (Gaiffe et Schmitt, 1980; Bruckert et Gaiffe, 1980; Michalet, 1982; Michalet et Bruckert, 1986), des relevés de la végétation et des descriptions de sols, incluant l'interface sol-roche, ont été effectués simultanément sur 300 stations selon la méthode sigmatiste (Guinochet, 1973) et en prenant pour référence la classification française des sols (CPCS, 1967). Les données, traitées par analyse factorielle des correspondances, ont conduit à définir les groupements végétaux et à sélectionner 30 sols sur lesquels ont été effectuées des analyses de caractérisation et les études indiquées cidessous.

Des observations ont été faites sur les caractéristiques des bancs : espacement, direction et densité des plans de fractures, dimension des fragments de roches, surface de contact solroche, dimension du système poral.

Des échantillons de calcaires prélevés dans les sols et dans la roche saine ont été soumis à des études microstructurales (sections fines), à des déterminations de la porosité par imbibition dans l'eau, à des analyses chimiques (éléments totaux) et minéralogiques (analyses RX par le laboratoire de géologie de Neuchâtel). Les résidus insolubles ont été obtenus par traitement $\mathrm{HCl}$ à $\mathrm{pH} 3$ contrôlé. Le taux de carbone des résidus insolubles a été déterminé par combustion (Carmograph). La matière organique a été analysée par pyrolyse (Tissot et al, 1974) au CNRS d'Orléans (URA 724). II s'agit d'une détection sélective des composés hydrocarbonés et de certains composés oxygénés $\left(\mathrm{CO}_{2}\right)$ libérés par 
la pyrolyse de la matière organique contenue dans un échantillon chauffé en programmation de température sous atmosphère contrôlée.

On obtient 4 pics, $S_{0}$ et $S_{1}$ représentant les gaz libérés à faible température, $S_{2}$ les composés hydrocarbonés et $S_{4}$ le $\mathrm{CO}_{2}$ libéré sous atmosphère oxydante. Le carbone organique total et défini par COT $=\left(0,82\left(S_{1}+S_{2}\right)+S_{4}\right) / 10$. Le pic $S_{2}$ permet de définir 2 parametres, la température maximale de craquage, qui indique le degré de "maturité" de la matière organique et l'indice d'hydrogène $\left(/ H=100 \mathrm{~S}_{2} / \mathrm{COT}\right)$, lié au rapport atomique $\mathrm{H} / \mathrm{C}$ et marquant le caractère aliphatique. Les 2 valeurs $T_{\text {max }}$ et $I H$ permettent d'établir un diagramme caratérisant les formes organiques d'origine marine, lacustre ou terrestre (Espitalie et al, 1977).

Les régimes hydriques de 2 sols ont été étudiés par des mesures de conductivité hydraulique (Ks) effectuées en conditions saturées dans des tubes d'acier enfoncés jusqu'à la roche, par des déterminations d'humidité volumique et par des mesures tensiométriques selon les techniques de Feodoroff et Ballif (1969).

L'organisation et la porosité structurale des sols ont été étudiées par l'observation de sections fines de grande taille $(2 \times 16 \mathrm{~cm})$. Les échantillons non perturbés et secs à l'air ont été imprégnés avec une résine de polyester avant fabrication des sections fines, effectuée à l'École nationale supérieure agronomique de Rennes.

\section{RÉSULTATS ET DISCUSSION}

\section{Caractéristiques et propriétés des bancs}

La morphologie des 3 types de bancs calcaires les plus répandus dans la HauteChaîne du Jura est présentée par les figures 1-3. Les clichés montrent clairement les différences très significatives qui caractérisent les fracturations et la géométrie des fragments de roche, relatives à chaque type. Bien que la variabilité des paramètres mesurés soit grande, le nombre des observations et la précaution d'étudier les bancs à la fois en coupe et en section ont permis de parvenir à un estimation approchée acceptable des caractéristiques des bancs (tableau I).

Les lapiez, qui occupent dans plusieurs secteurs des surfaces étendues (forêt du Massacre, Crêt de la Neige par exemple), apparaissent constitués d'un ensemble de blocs parallélépipédiques de plusieurs $\mathrm{m}^{3}$, séparés par de grandes fractures verticales espacées et largement ouvertes. Le système poral se compose de vides de très grande dimension. La surface de contact sol-roche est limitée à la face horizontale supérieure des bancs.

Les bancs concassés sont éclatés dans leur masse et sur plusieurs mètres d'épaisseur en cailloux et pavés cubiques de 5 à $15 \mathrm{~cm}$ de côté, comme permet de l'observer la carrière de la route forestière du Noirmont, près de Mouthe, dont le front de taille atteint en hauteur une vingtaine de $\mathrm{m}$. Les bancs concassés sont très répandus dans les massifs du Risol et du Risoux. Les fractures forment un réseau serré de plans orthogonaux, horizontaux et verticaux, avec une densité moyenne de 30 fractures par $m^{2}$, selon la dimension des cubes. Le système poral est très développé, bien que caractérisé par des espaces fins $\left(0,1-1 \cdot 10^{-2} \mathrm{~m}\right)$. La surface de contact sol-roche devient très étendue dans ce type de station.

Les dalles caractérisent les bancs fracturés selon des plans verticaux espacés, avec une densité moyenne de 6 fractures par $\mathrm{m}^{2}$. Les fragments parallélépipédiques de grande dimension sont séparés par des espaces fins à moyens mais au lieu d'être vides comme dans les lapiez et les calcaires concassés, ces espaces sont remplis de résidus insolubles, constituants alumino-silicatés libérés par altération des bancs. La surface de contact sol-roche est limitée à la face supérieure des bancs. 


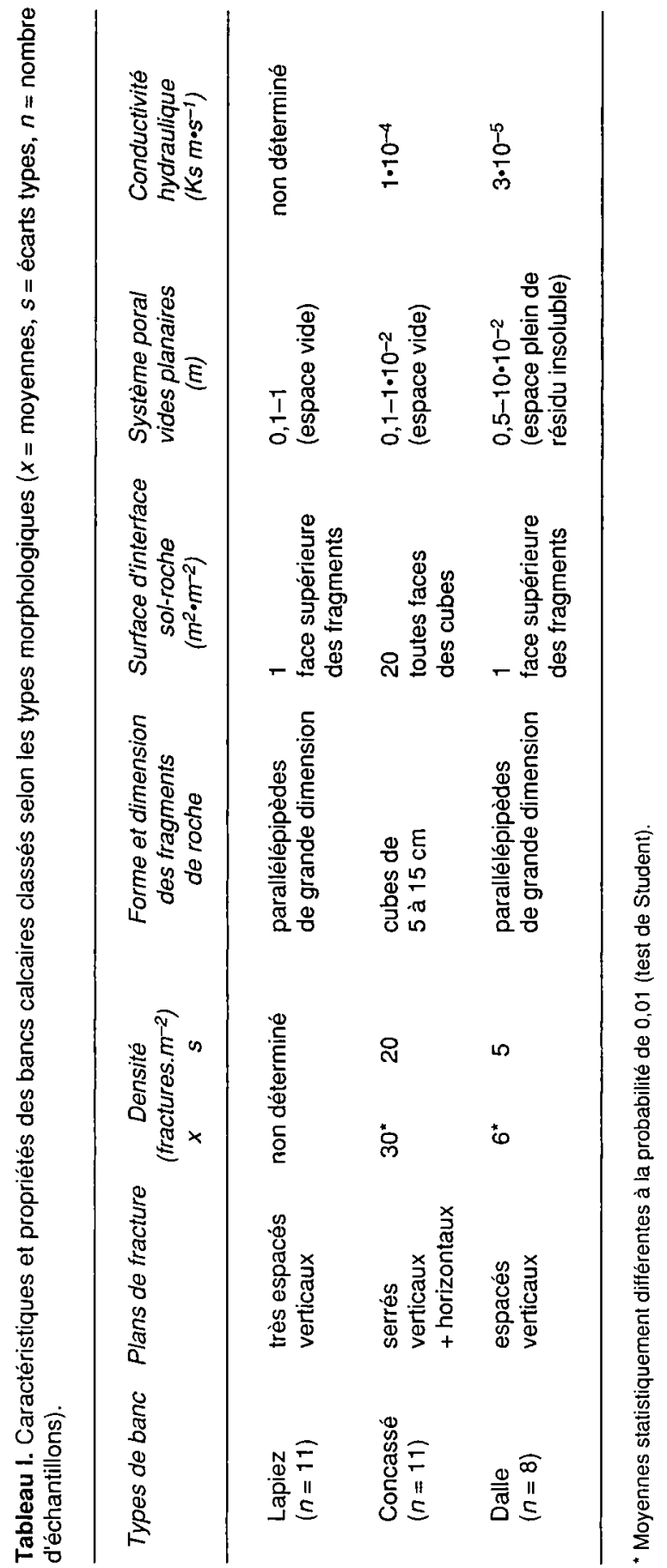




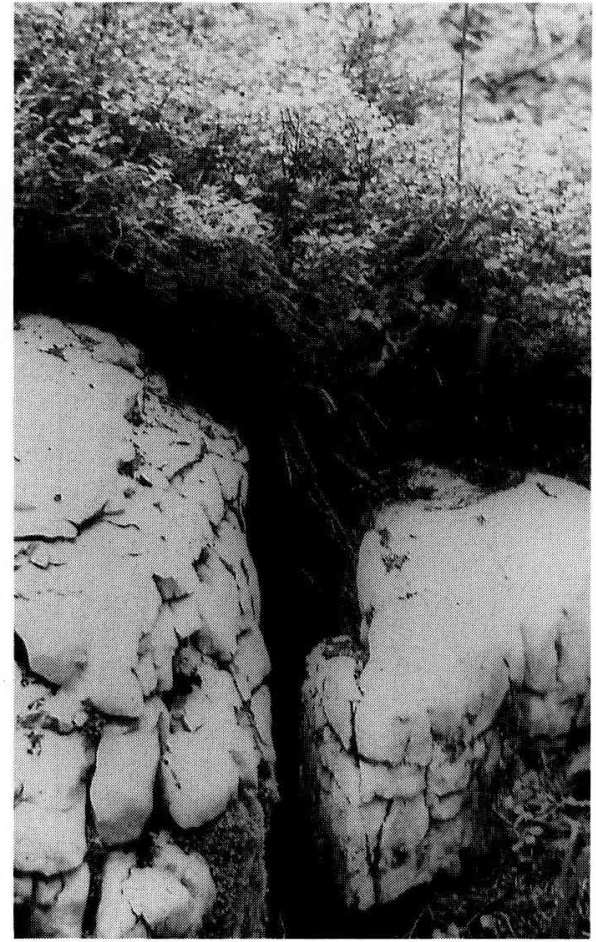

Fig 1. Sol litho-calcique sur lapiez.

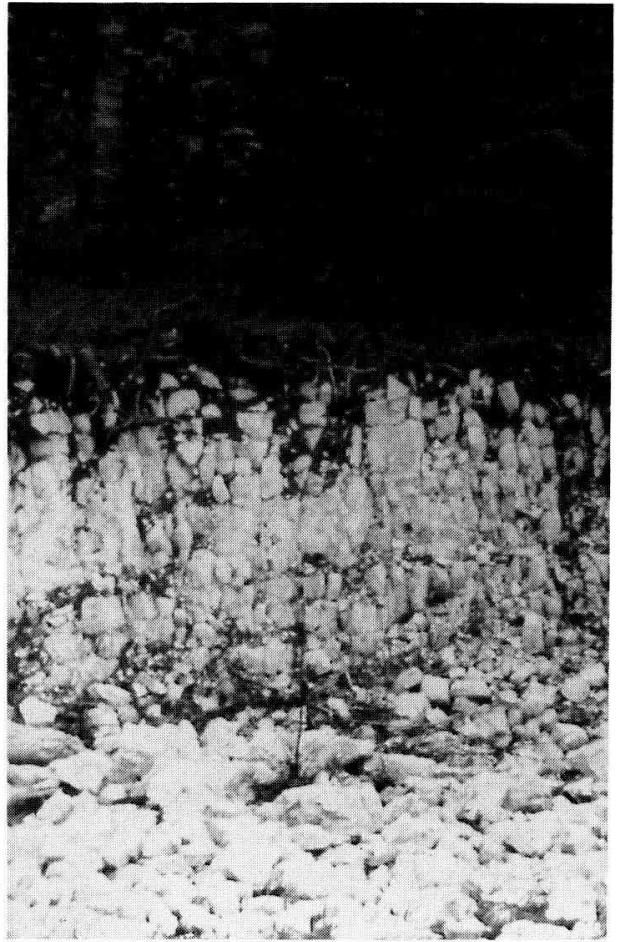

Fig 2. Banc calcaire concassé (fractures fines en réseau serré).

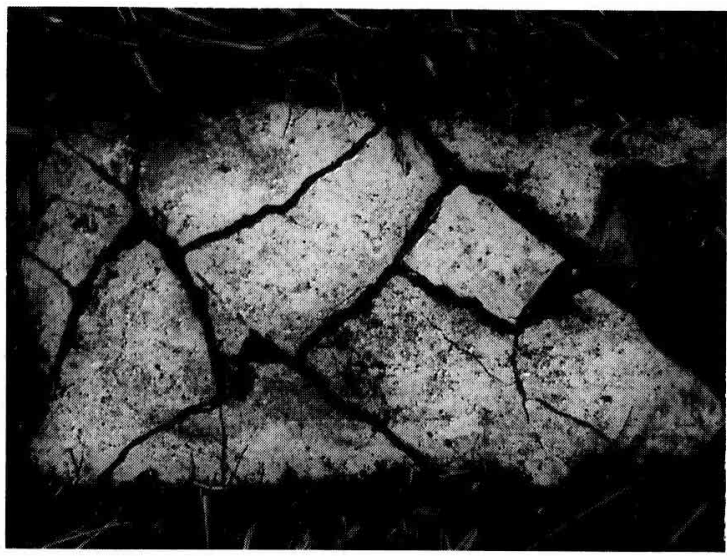

Fig 3. Dalle vue de dessus. 
On n'a aucune information sur les densités de fracturation à l'échelle des bancs rencontrés dans la Haute-Chaîne. Itty et Vintaer (1987) qui ont étudié la région, se sont attachés à déterminer les directions principales prises par les fractures. On connaît par contre des densités de fracturation sur les premiers plateaux (Forestier et Verez, 1978; Salperwyck, 1990). Les résultats obtenus ont démontré l'extrême variabilité des densités observées d'un site à l'autre, ces derniers étant pris au hasard des affleurements et des falaises. Par contre, la sélection d'un type de banc - à savoir la dalle de la carrière de Briod sur le plateau lédonien - a permis à Salperwick (1990) d'obtenir des valeurs plus resserrées allant de 2 à 5 fractures par $\mathrm{m}^{2}$, en accord avec nos propres observations.

\section{Groupements végétaux et sols}

Les groupements végétaux propres aux 3 types de bancs définis ci-dessus sont parfaitement caractérisés. Ils subissent, en fonction de l'altitude, une évolution qui permet de définir sur des bases statistiques (Schmitt, 1980; Michalet, 1982), des groupements différents selon les étages bioclimatiques. C'est ainsi qu'aux étages montagnard et subalpin du Jura, entre 850 et $1700 \mathrm{~m}$ d'altitude, la végétation seminaturelle des positions hautes ou convexes se répartit en 9 groupements (tableau II). Ils appartiennent aux alliances du Fagion et du Vaccinio-Piceion, mais aussi aux groupements de pelouses du Seslerion qui occupent certaines situations de l'étage subalpin dans lesquelles Beguin (1972) a démontré que la forêt ne pouvait pas s'implanter pour des raisons mécaniques (effet structural et effet culminal).

Sur les lapiez se développent, selon l'altitude :
- à l'étage montagnard inférieur (850$1100 \mathrm{~m}$ ), la pessière à doradille (AsplenioPiceetum Kuoch 54) dans sa forme appauvrie, sans listère cordée ni lycopode;

- à l'étage montagnard supérieur (1 100$1400 \mathrm{~m}$ ), la pessière à doradille dans sa forme typique;

- à l'étage subalpin (au-dessus de $1400 \mathrm{~m}$ ), la pineraie à lycopode (Lycopodio-Mugetum Richard 61), seul groupement forestier de cet étage dans le Jura (Beguin, 1972; Michalet, 1982).

Les calcaires concassés, fortement drainants, supportent :

- jusque vers $1100 \mathrm{~m}$, la hêtraie à dentaire (Dentario-Fagetum Moor $52 \mathrm{~T}$ Muller 67);

- de 1100 à $1400 \mathrm{~m}$ environ, la hêtraie à érable dans sa variante "xérophile" à sol humifère (Aceri-Fagetum melampyretosum Michalet 80);

- dans l'étage subalpin, au-delà de $1400 \mathrm{~m}$, un groupement non forestier, parfois à aspect de lande (cotoneaster, genévrier, raisin d'ours), appartenant aux pelouses du Seslerio-Arctostaphyletum (Faure 68 Beguin 71).

Sur les calcaires en dalles, on trouve :

- la forme modale de la hêtraie-sapinière (Abieti-Fagetum Bartsch 40), dans une large tranche altitudinale comprise entre 850 et $1200 \mathrm{~m}$;

- la hêtraie à érable (Aceri-Fagetum typicum Moor 52) à l'étage montagnard supérieur, jusque vers $1400 \mathrm{~m}$;

- des pelouses subalpines à seslérie et laîche (Seslerio-Caricetum sempervirentis $\mathrm{Br} \mathrm{Bl}$ et Jenny 26) au-dessus de $1400 \mathrm{~m}$, dans la zone où l'implantation de la forêt est impossible faute d'ancrage suffisant pour les arbres.

Les 3 modes de fracturation des roches qui déterminent l'existence des groupe- 


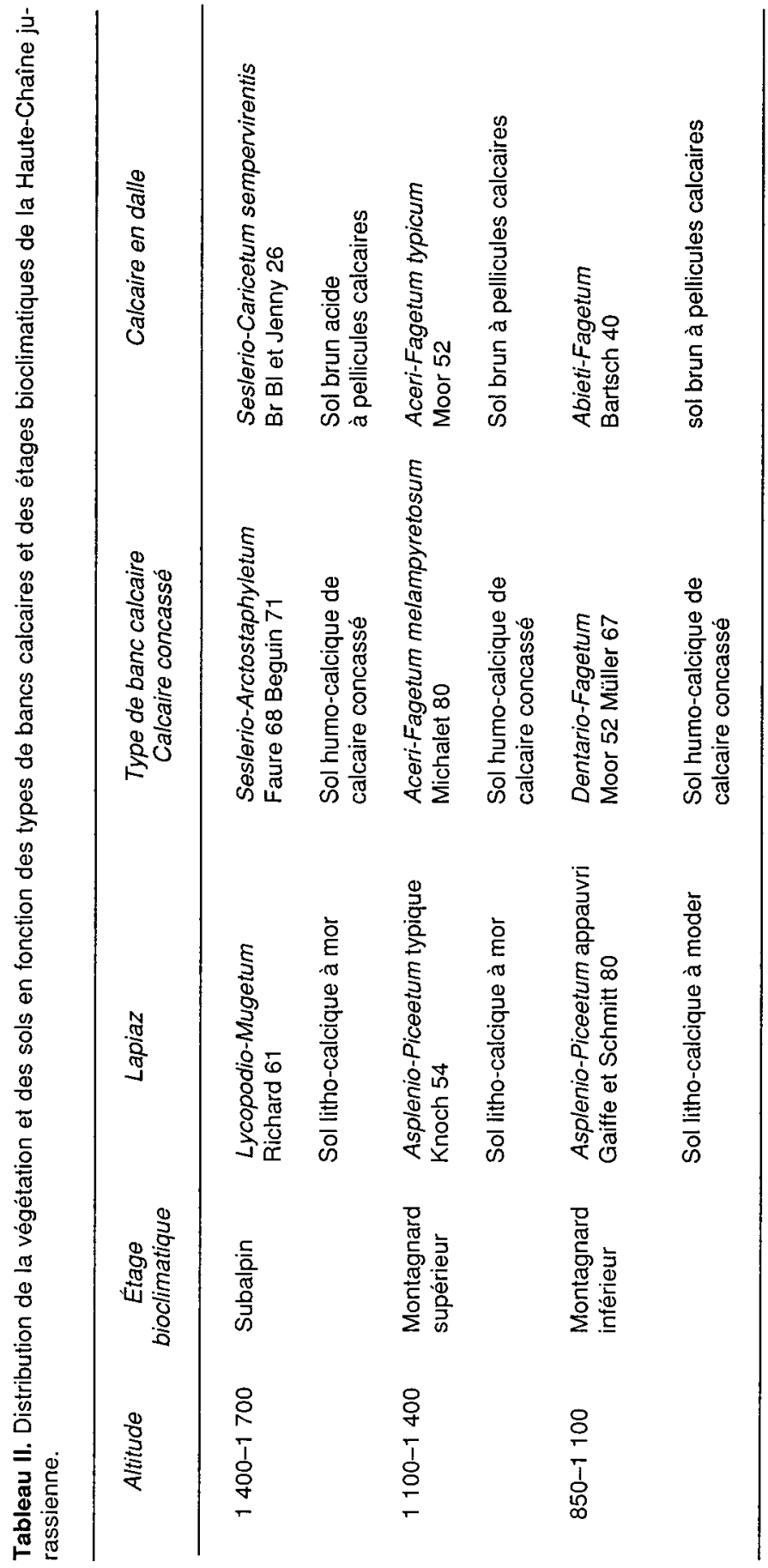


ments végétaux conduisent également à 3 types de sols (tableau II et fig 4) très différents dans leurs caractéristiques analytiques (tableau III) et leurs processus de pédogenèse et de fonctionnement.

Les sols litho-calciques à mor liés aux lapiez sont constitués d'un horizon épais organique fibreux, rougeâtre sombre et d'un horizon granuleux noir rougeâtre (5 YR 3/3 couleur Munsell à l'état sec) reposant sur la roche. Cet humus très acide est colonisé par des racines d'éricacées (Vaccinium myrtillus).

Les sols humo-calciques liés aux bancs concassés sont constitués d'un complexe argilo-humique brun très sombre ( 5 YR $2,5 / 2)$ organisé en agrégats très stables mélangés à des cailloux calcaires durs. La forte capacité d'échange $(60 \mathrm{me} / 100 \mathrm{~g})$ est saturée, notamment en calcium, et le $\mathrm{pH}$ reste proche de la neutralité.

Les sols bruns à pellicules calcaires des dalles comprennent un horizon $A_{1}$ brun foncé (10 YR 3/4) et un horizon (B) brun (10 YR 3/3), structurés en agrégats polyédriques. Ils reposent sur la dalle compacte avec, pour transition, des pellicules d'arrachement calcaires. La terre fine, partiellement désaturée et modérément acide à l'étage montagnard, devient fortement désaturée $(30-50 \%)$ et très acide $(\mathrm{pH} 5)$ à l'étage subalpin.

Toutes ces observations d'ordre morphologique et analytique, effectuées sur l'ensemble des sols étudiés, attirent l'attention sur les points suivants :

- sur roches très drainantes, largement ouvertes (lapiez) ou abondamment fracturées
Sol

litho-calcique sur lapiaz
Sol

humo-calcique sur banc concossé
Sol brun d̀ pellicules calcaires sur dalle
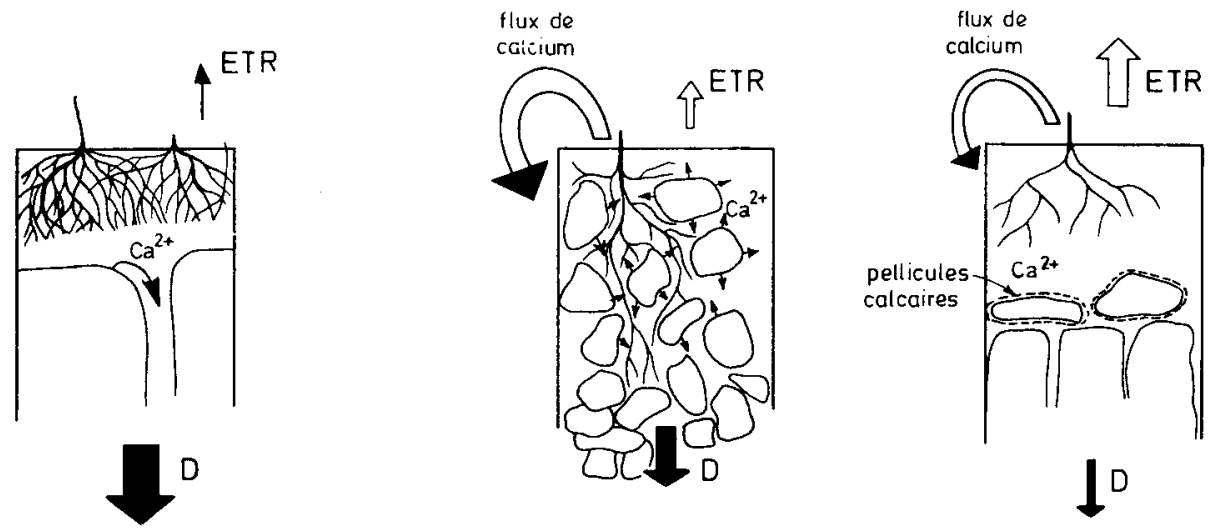

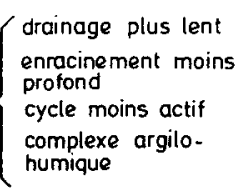

Fig 4. Morphologie des profils, flux hydriques et flux de calcium dans les 3 stations. ETR : évapotranspiration réelle; $\mathrm{D}$ : drainage. 


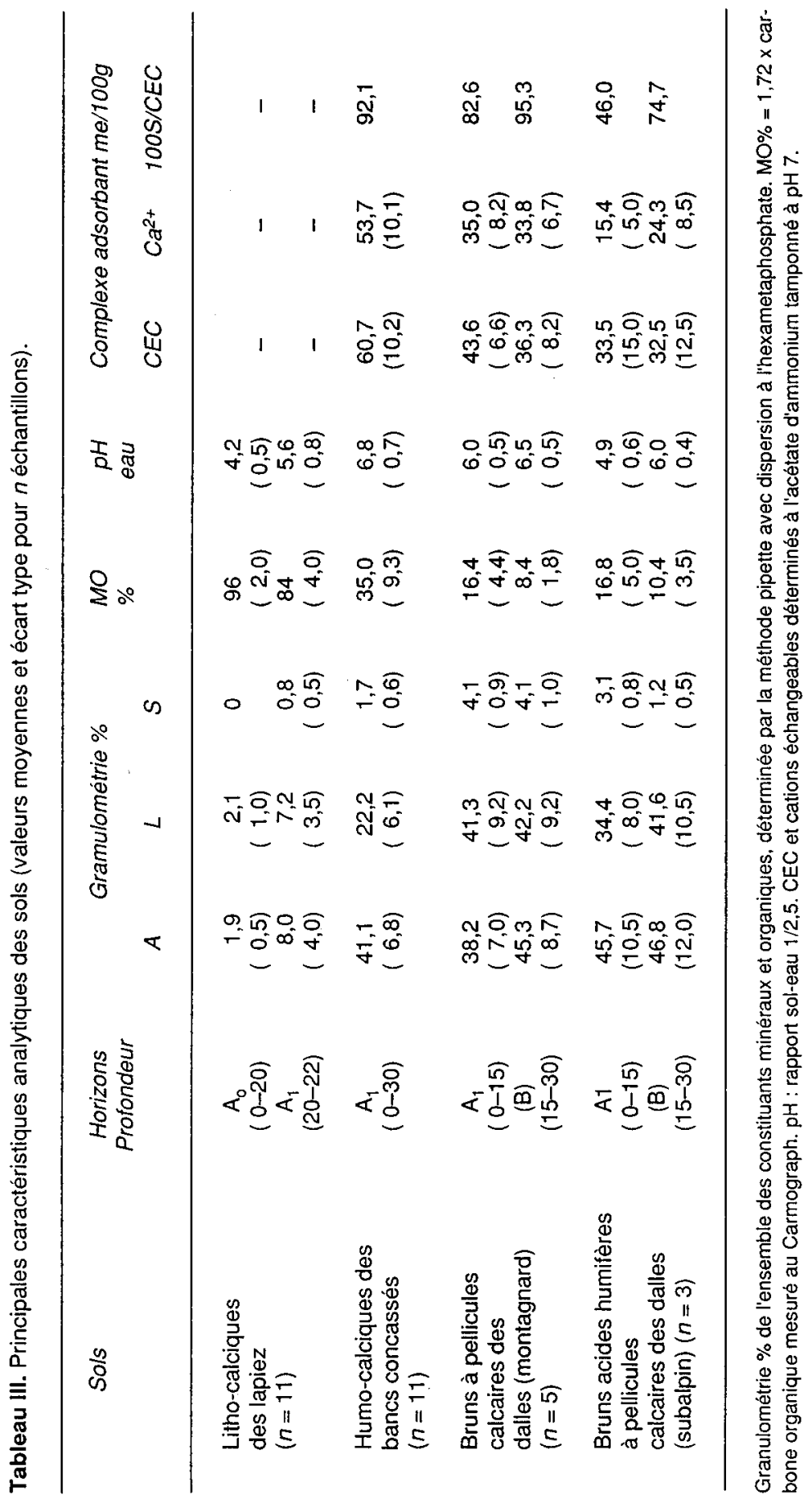


(calcaires concassés), les sols sont très humifères mais, en fonction de la taille et du mode de distribution des cailloux dans les profils, la nature des humus diverge totalement : mor désaturé très acide d'un côté, mull saturé en calcium de l'autre;

- sur roches modérément drainantes (dalles), les sols sont significativement moins humifères.

Des statuts organiques aussi différents traduisent des modes de décomposition ainsi que des processus pédogénétiques et fonctionnels très différents.

\section{Transferts hydriques et processus de pédogenèses}

Les fractures agissent comme des chenaux le long desquels la circulation des eaux météoriques est rapide, avec peu de flux d'eau parvenant à pénétrer dans les matrices adjacentes formées de roches de faible porosité (Grisak et Pickens, 1980). Des tests effectués sur une dizaine d'échantillons ont montré que les matrices des calcaires étudiés ici étaient imperméables, leur taux d'imbibition n'étant que de $0,8 \% \pm 0,3$. La porosité "de moulage» ou intragranulaire qui les caractérise n'est que peu accessible à l'eau.

Le système poral des bancs calcaires considérés assure l'évacuation des eaux gravitaires vers les cavités et les circulations souterraines du karst. Cependant, les transferts hydriques se font à travers les sols et le réseau poral sous-jacent, avec des dynamiques et des effets variables selon les caractéristiques et les propriétés des bancs (fig 4).

Dans les lapiez, l'eau des précipitations s'engouffre de façon immédiate dans les larges fractures, entrainant avec elle les produits de dissolution formés à l'interface entre le mor agressif et les blocs calcaires. Ces derniers ne sont attaqués qu'en surface et ne libèrent que très peu de bicarbonate de calcium et d'argiles (résidu inso- luble) immédiatement exportés dans le réseau karstique. Les remontées de calcium par le cycle biologique sont quasi nulles. II en résulte que le mor reste très acide $(\mathrm{pH}$ 4) et que le milieu demeure très hostile à la microflore minéralisatrice : la matière organique non décomposée s'accummule ( $\mathrm{MO}=96 \%$ ). L'absence d'argile interdit la constitution de complexes organominéraux.

Avec les bancs concassés, la conductivité hydraulique mesurée en régime saturé (Ks) à l'interface sol-roche, est très grande $\left(1 \cdot 10^{-4} \mathrm{~m} \cdot \mathrm{s}^{-1}\right.$ tableau 1). Dans les sols, les variations de potentiel hydrique avec le temps sont étroitement synchrones avec la pluviométrie et se caractérisent par des écarts de forte amplitude (fig 5) démontrant que les sols subissent des alternances répétées d'humectationdessiccation. Ces processus sont à l'origine d'une importante porosité structurale dans les matériaux pédologiques. En section fine (fig 6), le plasma apparaît frag-

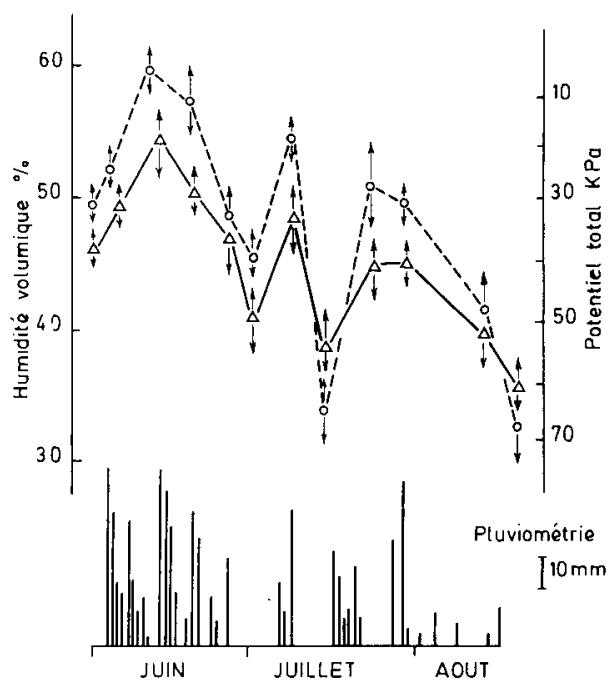

Fig 5. Régime hydrique en saison estivale dans un sol humo-calcique : variations alternées d'humectation-dessiccation en relation avec la pluviométrie. Prélèvements à $15 \mathrm{~cm}$ (ronds) et à $30 \mathrm{~cm}$ (triangles). 
menté selon un réseau polygonal résultant de fentes de retrait et de l'organisation de polyèdres. Ces vides sont interconnectés à de nombreuses cavités et chenaux d'origine biologique dans lesquels on reconnaît d'abondants agrégats arrondis fabriqués par les vers de terres. La répartition régulière des cailloux calcaires dans les profils favorise le maintien d'une ambiance calcique (fig 4), à l'origine de la formation d'un complexe argilo-humique biologiquement et physiquement très stable (Gaiffe et al, 1984; Gaiffe, 1987).

Avec les dalles, la conductivité hydraulique apparaît nettement inférieure à celle des bancs concassés $\left(3 \cdot 10^{-5} \mathrm{~m} \cdot \mathrm{s}^{-1}\right)$, mais demeure largement suffisante pour assurer l'évacuation encore rapide des eaux gravitaires. Les processus de structuration et d'organisation du plasma des sols restent toujours très actifs (fig 7). Les sites de dissolution du calcaire étant limités à la surface plane de contact sol-banc, l'ambiance calcique et les remontées biologiques sont de ce fait diminuées (fig 4), ce qui entraine une baisse de la stabilisation biologique des composés humiques dans les complexes argilo-humiques (Gaiffe et al, 1984).

Ainsi, dans des milieux qui reçoivent les mêmes quantités de précipitations, le substratum géologique - par le biais de la fracturation des roches, de la géométrie des fragments et de l'organisation à l'interface sol-roche - crée des flux hydriques et des flux calciques qui différencient les principaux écosystèmes du Jura karstique aux étages montagnard et subalpin.

\section{Analyse des calcaires}

Plusieurs caractéristiques analytiques différencient les roches considérées : ce sont
6

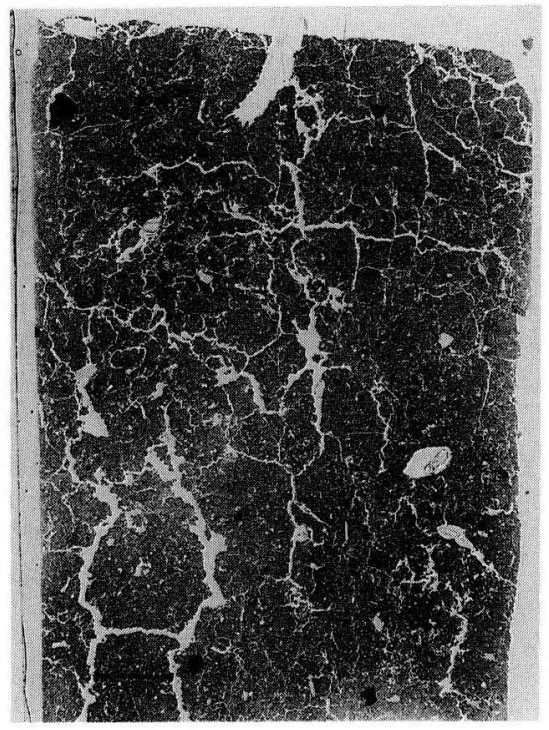

7

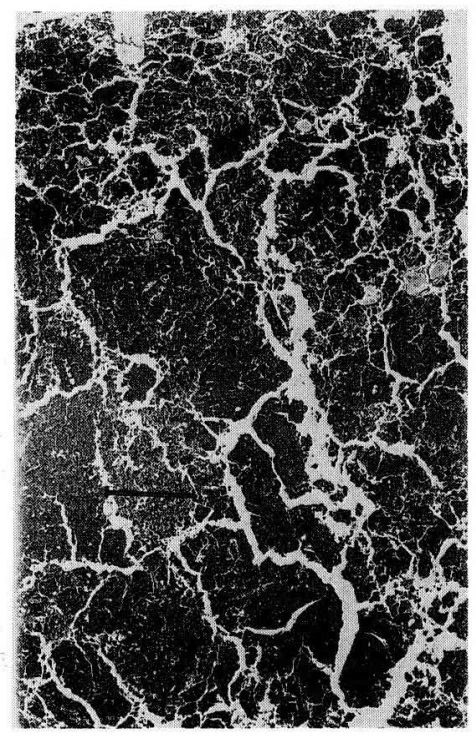

Fig 6 et 7. Structuration d'origine physique et biologique dans les horizons A. Fig 6 : sol humocalcique; Fig 7 : sol brun à pellicules calcaires (les traits représentent $1 \mathrm{~cm}$ ). 
la calcite, la dolomie, le résidu insoluble et la matière organique de ce résidu. Les lapiez et les calcaires concassés ont une composition minérale constituée presque exclusivement de calcite pure. Par contre, les calcaires en dalle renferment beaucoup de dolomie et significativement plus de quartz, d'argiles et d'oxydes (tableau IV). La variabilité des échantillons apparaît très faible pour les premiers et, à l'inverse, très élevée pour les seconds (écarts types forts).

Les résidus insolubles (tableau $V$ ) qui regroupent le quartz, les argiles, les oxydes et de la matière organique, discriminent de façon très significative les 3 types de bancs : les calcaires concassés se distinguent par des résidus extrêmement réduits $(0,7 \%)$, suivis de près par les lapiez $(1,3 \%)$. Les dalles rassemblent les calcaires les moins purs $(4,9 \%)$.

Le carbone organique varie de façon inversement proportionnelle au résidu insoluble (fig 8 ), en raison d'un effet de dilution, mais aussi parce que de la matière organique en solution a été associée à la précipitation des carbonates (Bonnefous et Kubler, 1968, Trichet, 1968) pendant la sédimentation. Valles et al (1989) ont mon- tré que la complexation calcium-molécules organiques en solution interfère dans les équilibres géochimiques des systèmes $\mathrm{CaCO}_{3}-\mathrm{CO}_{2}-\mathrm{H}_{2} \mathrm{O}$. II s'ensuit que les différences de taux moyens en carbone organique dosés dans les résidus insolubles (tableau V) sont très significatifs pour différencier les lapiez des calcaires concassés.

En portant graphiquement les indices d'hydrogène en fonction des températures maximales de crackage (fig 9), les points obtenus se situent tous dans le secteur regroupant les échantillons organiques d'origine marine (Tissot et al, 1974). Ce résultat étaye l'interprétation proposée cidessus d'une co-précipitation de substances organiques et de carbonates lors de la sédimentation.

II ressort de l'ensemble des résultats analytiques portant sur les calcaires, que la différenciation des bancs s'est opérée précocement, sous la dépendance des conditions d'environnement au moment de la sédimentation. Ces dernières sont à l'origine de la composition variée des roches (Purser, 1980) et, par voie de conséquence, de leurs aptitudes mécaniques différentes. Les phases tectoniques qui ca-

Tableau IV. Étude minéralogique des roches classées par types de bancs : minéraux déterminés par spectographie $\mathrm{RX}$ et analysés chimiquement $(\bar{x}=$ moyennes en $\%, s=$ écarts types, $n=$ nombre d'échantillons).

\begin{tabular}{|c|c|c|c|c|c|c|}
\hline \multirow{2}{*}{$\begin{array}{c}\text { Minéraux } \\
\text { de la roche } \\
\%\end{array}$} & \multicolumn{2}{|c|}{$\begin{array}{l}\text { Lapiez } \\
n=11\end{array}$} & \multicolumn{2}{|c|}{$\begin{array}{c}\text { Calcaires concassés } \\
\qquad n=11\end{array}$} & \multicolumn{2}{|c|}{$\begin{array}{c}\text { Dalles } \\
n=8\end{array}$} \\
\hline & $\bar{x}$ & $s$ & $\bar{x}$ & $\mathrm{~s}$ & $\bar{x}$ & s \\
\hline Calcite & $98,7(a)$ & 1,6 & $98,9(a)$ & 3,9 & $43,5(b)$ & 20,3 \\
\hline Dolomie & 0,0 & - & $0,3(a)$ & 0,3 & $51,6(b)$ & 16,8 \\
\hline Quartz & 0,4 (a) & 0,2 & $0,5(a)$ & 0,2 & $2,2(a)$ & 2,2 \\
\hline Argiles, oxydes & 0,9 (a) & 0,3 & 0,3 (a) & 0,2 & $2,7(b)$ & 1,7 \\
\hline
\end{tabular}

Les mêmes lettres dans les lignes signifient que les moyennes ne sont pas statistiquement différentes à la probabilité de 0,01 (test de Student). 
Tableau V. Teneur en résidu insoluble des roches classées par type de bancs et analyse des substances organiques du résidu insoluble ( $\bar{x}=$ moyennes, $s=$ écarts types, $n=$ nombre d'échantillons).

\begin{tabular}{|c|c|c|c|c|c|c|}
\hline & \multicolumn{2}{|c|}{$\begin{array}{l}\text { Lapiez } \\
n=11\end{array}$} & \multicolumn{2}{|c|}{$\begin{array}{c}\text { Calcaires concassés } \\
\quad n=11\end{array}$} & \multicolumn{2}{|c|}{$\begin{array}{l}\text { Dalles } \\
n=8\end{array}$} \\
\hline & $\bar{x}$ & $s$ & $\bar{x}$ & $s$ & $\bar{x}$ & $s$ \\
\hline $\begin{array}{l}\text { Résidu insoluble } \\
\% \text { de la roche }\end{array}$ & $1,3(a)$ & 0,4 & 0,7 (b) & 0,4 & $4,9(c)$ & 1,7 \\
\hline $\begin{array}{l}\mathrm{C} \text { organique } * \% \text { du } \\
\text { résidu insoluble }\end{array}$ & $3,2(a)$ & 0,8 & $8,0(b)$ & 2,7 & $1,7(c)$ & 1,2 \\
\hline $\mathrm{T}^{\circ} \mathrm{C}$ maximale ${ }^{* *}$ & 421,0 & 16,0 & 428,0 & 5,0 & 415,0 & 14,0 \\
\hline$S_{2}^{* *}$ & 11,0 & 2,3 & 32,2 & 6,8 & 5,6 & 4,6 \\
\hline $\begin{array}{l}\text { Indice hydrogène }{ }^{\star *} \\
100 S_{2} / C \text { organique }\end{array}$ & 343,0 & 81,0 & 404,0 & 91,0 & 332,0 & 24,0 \\
\hline
\end{tabular}

Les mêmes lettres dans les lignes signifient que les moyennes ne sont pas statistiquement différentes à la probabilité de 0.01 (test de Student). " $: C$ organique dosé au Carmograph; ** : Analyse par pyrolyse; $\mathrm{S}_{2}:$ Composés hydrocarbonés.

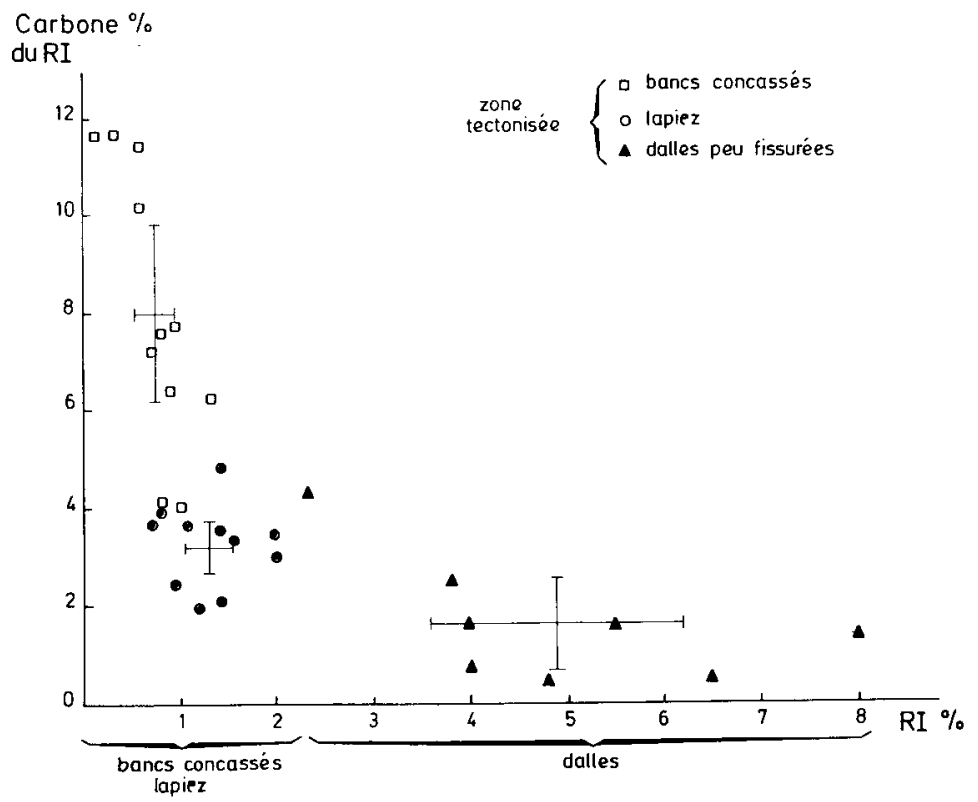

Fig 8. Distribution des roches en fonction du taux de résidu insoluble (RI \%) et de sa richesse en carbone ( $\mathrm{C} \%$ du $\mathrm{Rl})$. Valeurs moyennes et erreur standard à la moyenne $(P=0,05)$. 


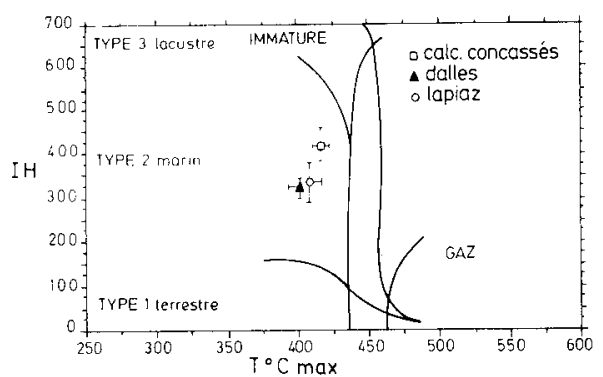

Fig 9. Diagramme des résidus insolubles des 3 types de calcaires (pyrolyse Rock-Eval) : indices d'hydrogène $\mathrm{IH}$ en fonction des températures maximales de crackage.

ractérisent l'orogenèse jurassienne ont ultérieurement révélé les potentialités inscrites dans les roches. On peut donc attribuer la morphologie actuelle des bancs à l'enchaînement de 2 séries d'événements majeurs, les premiers - dans la chronologie comme dans l'importance - étant de nature sédimentologique et les deuxièmes de nature orogénique.

\section{CONCLUSION}

La diversité du cortège floristique climacique aux étages montagnard et subalpin du Jura apparaît comme un révélateur très sensible des conditions de milieu. Le gradient climatique entraîne des changements du cortège floristique avec l'altitude et se traduit également par l'existence d'un étage subalpin pratiquement asylvatique (Flahaut, 1901) en raison d'un fort effet culminal (Béguin, 1972).

Cependant, à un étage bioclimatique donné, c'est le niveau trophique qui produit la diversification. Celui-ci, lié aux flux d'eau et de matières qui sont spécifiques de chaque unité pédologique, se trouve être la ré- sultante non pas des seules propriétés du sol proprement dit mais du fonctionnement d'un système de subsurface dans lequel la roche sous-jacente exerce une action déterminante par l'intermédiaire de son réseau poral (Bruckert et Gaiffe, 1990). D'où l'importance, pour l'interprétation des paysages botaniques et pédologiques, de considérer la géométrie de la roche-mère et son niveau de perméabilité.

Comme le montre cette étude, les types de sédimentation qui ont contribué au déterminisme chimique des calcaires jurassiens ont, par la suite, induit les réponses des roches, des sols et des plantes. II s'est établi, entre les paléo-environnements du bassin sédimentaire de l'ère secondaire et

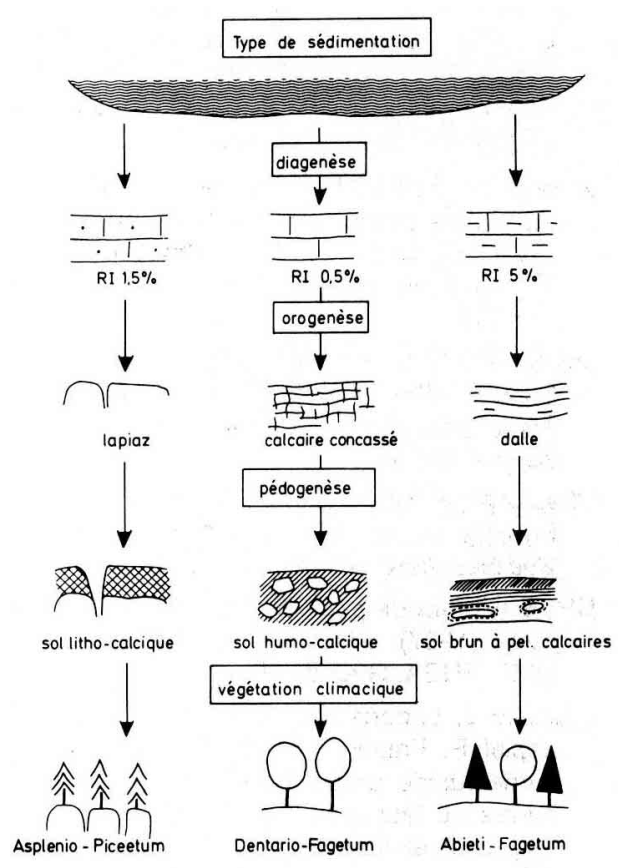

Fig 10. Exemples de dépôts de la plate-forme jurassique, à l'origine de calcaires durs. Relations entre l'écologie du bassin sédimentaire et la végétation climacique actuelle (étage montagnard). 
les associations végétales actuelles, une filiation (fig 10) qui fait remonter à 150 millions d'années les causes de la différenciation des écosystèmes.

\section{RÉFÉRENCES}

Aubert D (1969) Phénomènes et formes du karst jurassien. Eclogae Geol Helv 62, 325399

Béguin C (1972) Contribution à l'étude phytosociologique et écologique du Haut-Jura. Thèse sciences, univ de Neuchâtel, $190 p$

Bonnefous J, Kubler B (1968) Étude stratigraphique et géochimique d'échantillons de terrains jurassiques de Tunisie (Région NordEst). Bull Centr Rech Pau, SNPA, 283-320

Bruckert S, Gaiffe M (1980) Analyse des facteurs de formation et de distribution des sols en pays calcaire glaciaire ou karstique. Plaine de Frasne-Bonnevaux et Montagne du Laveron, Jura Central. Ann Sci Univ Besançon Biol Vég 4, 1, 19-65

Bruckert S, Gaiffe M (1990) Les systèmes de subsurface sols-roche, modèles de pédogénèses. Exemples des domaines karstique et non karstique de Franche-Comté. Sci Sol 28 , 319-332

Campy M (1982) Le quaternaire franc-comtois. Essai chronologique et paléoécologique. Thèse doct état, univ Franche-Comté - Besançon, $575 p$

Chauve $P$ (1990) Évolution du Jura externe de Franche-Comté au cours du tertiaire. Bull Soc Neuchâtel Sci Nat 113, 27-39

CPCS (Comm de pédologie et de cartographie sols) (1967) Classification des sols. Édn 1967, ENSA, Grignon, $97 p$

Espitalié J, Laporte JL, Madec M, Marquis F, Leplat P, Paulet J, Boutefeu A (1977) Méthode rapide de caractérisation des roches mères de leur potentiel pétrolier et de leur degré d'évolution. Rev Inst Fr Pétrole 32, 2341

Feodoroff J, Ballif JL (1969) Étude de l'infiltration de la pluie in situ à l'aide du tensiomètre. Ann Agron 20, 457-504
Flahaut C (1901) Les limites supérieures de la végétation forestière et les prairies pseudoalpines en France. Rev Eaux et Forêts XL

Forestier G, Verez C (1978) Étude de la fracturation du plateau d'Ornans. Ann Sci Univ Besançon Géologie 29, $3^{e}$ sér, 97-103

Gaiffe M (1987) Processus pédogénétiques dans le karst jurassien. Analyse de la complexation organo-minérale en ambiance calcique. Thèse doct état, univ Franche-Comté Besançon, $160 \mathrm{p}$

Gaiffe M, Bruckert S (1985) Analyse des transports de matières et des processus pédogénétiques impliqués dans les chaînes de sols du karst jurassien. Soils and Geomorphology, Catena ( $n^{\circ}$ spécial) 6, 159-174

Gaiffe $M$, Schmitt A (1980) Sols et végétation à l'étage montagnard dans les forêts du Jura Central. Sci Sol 4, 265-296

Gaiffe M, Duquet B, Tavant H, Tavant $Y$, Bruckert S (1984) Stabilité biologique et comportement physique d'un complexe argilohumique placé dans différentes conditions de saturation en calcium ou en potassium. Plant and Soil 77, 271-284

Grisak GE, Pickens JF (1980) Solute transport through fractured media. I. The effect of matrix diffusion. Water Resour Res 16, 719

Guinochet M (1973) Phytosociologie. Masson, Paris, $277 p$

Itty $J$, Vintaer $J$ (1987) Contribution à l'étude géologique et hydrologique du haut bassin de l'Ain (Jura). Thèse doct $3^{\theta}$ cycle, univ Franche-Comté - Besançon, $290 \mathrm{p}$

Lamouroux $M$ (1972) Étude de sols formés sur roches carbonatées. Pédogenèses fersiallitiques au Liban. Thèse doct état, univ Strasbourg, Mémoire Orstom, Paris, 56, 266 p

Mathieu D, Petiot R (1973) Géodynamique externe. Ettude intégrée du milieu naturel. Rev Géomorphol Dyn 4, 179-187

Michalet R (1982) influence du climat général sur l'évolution des sols à l'étage subalpin dans le Jura. Thèse doct $3^{e}$ cycle, biol et physiol vég, univ Nancy I et Franche-Comté Besançon, $105 p$

Michalet R, Bruckert S (1986) La podzolisation sur calcaire à l'étage subalpin du Jura. Sci Sol 24, 363-374 
Purser BH (1980) Sédimentation et diagenèse des carbonates néritiques récents. Les éléments de la sédimentation et de la diagenèse. Institut Français du Pétrole, École Nationale Supérieure du Pétrole et des Moteurs, tome 1, $366 p$

Salperwyck M (1990) Structure et réponses hydrochimiques d'un système karstique de plateau (bassin des reculées de la Seille, Jura). Thèse doct $3^{e}$ cycle, univ Franche-Comté Besançon, $228 \mathrm{p}$

Thurmann J (1849) Essai de phytostatique appliquée à la chaîne du Jura et aux contrées voisines, ou étude de la dispersion des plantes vasculaires envisagée principalement quant à l'influence des roches sous-jacentes. Jent et Gassmann, Berne et Salerne, 2 vol, 444 et $373 p$
Tissot B, Espitalié J, Deroo G, Tempere C, Jonathan $D$ (1974) Origine et migration des hydrocarbures dans le Sahara oriental (Algérie). In: Advances in Organic Geochemistry. (B Tissot, F Bienner, ed) Technip, Paris, 315334

Trichet J (1968) Étude de la composition de la fraction organique des oolites. Comparaison avec celle des membranes des bactéries et des cyanophycées. CR Acad Sci Paris, 267, D, 1492-1494

Valles V, Bernadac A, Tardy $Y$ (1988) Étude géochimique de solutions riches en anions organiques. Application aux Chernozems. Cah ORSTOM Sér Pédol 24, 263-274

Villain J (1965) Contribution à l'étude de la gélivité des calcaires jurassiques en FrancheComté. Thèse doct $3^{e}$ cycle, Besançon, $86 p$ 